\title{
Giant Left Atrium - A Rare Case of Acute Upper Gastrointestinal Bleeding
}

\author{
Khus Raj Dewan, ${ }^{1}$ Bhanumati Saikia Patowary, ${ }^{1}$ Subash Bhattarai ${ }^{1}$ \\ 'Department of Gastroenterology, CMS-TH, Bharatpur, Nepal.
}

\section{ABSTRACT}

Acute upper GI bleeding is a common medical emergency with a hospital mortality of approximately $10 \%$. Non variceal UGI bleeding is the most common cause followed by oesophageal varices. Various rare causes have been described in the literature but there are very few cases of giant left atrium leading to oesophageal erosion and causing upper GI bleeding. We are presenting a case of rheumatic valvular heart disease with giant left atrium who presented in our department with acute upper GI bleeding.

Keywords: giant left atrium; oesophgeal erosion; upper GI bleeding.

\section{INTRODUCTION}

In mitral valvular heart disease, mostly mitral stenosis alone or with mitral insufficiency, the left atrium dilates and hypertrophies. Occasionally, the left atrium reaches extreme sizes as a result of increasing pulmonary artery pressure contributing to an increase in right side pressure and unfavorably affects morbidity and mortality rates due to complications like atrial fibrillation, heart failure, thromboembolism, pressure effects on surrounding structures producing dysphagia, causing erosions of the oesophagus etc. A giant left atrium can be assessed by a chest xray showing massive cardiomegaly with increased cardiothoracic ratio. Transthoracic echocardiography is the easiest method to diagnose giant left atrium.

\section{CASE REPORT}

A 54 year-old man, a farmer was admitted to College of Medical Sciences, Bharatpur, Nepal with chief complaints of epigastric pain along with passage of multiple episodes of black colored stool from 2 days. There was no history of blood vomiting, dysphagia or hoarseness of voice. He was a diagnosed case of rheumatic mitral valve disease since 34 years with
NYHA grade II. Penicillin was stopped many years back and currently was on diuretics and digoxin. He was a non smoker and didn't consume alcohol.

At the time of admission, he was conscious, alert, oriented to time place and person. General examination revealed moderate pallor with normal JVP. Vital signs revealed an irregular pulse $96 \mathrm{~b} / \mathrm{m}, \mathrm{BP} 110 / 70$ $\mathrm{mm} \mathrm{Hg}$ and temperature of $37^{\circ} \mathrm{C}$. In cardiovascular examination, apex beat was at $6^{\text {th }}$ ICS $4 \mathrm{~cm}$ lateral to left $\mathrm{MCL}$, hyperdynamic in nature. Left parasternal heave was present. On auscultation Grade 3 to 4 murmurs of severe MS, MR, TR were heard without any evidence of congestive cardiac failure or subacute bacterial endocarditis.. Chest examination showed mild bilateral fine basal crepitation. Other systemic examinations were unremarkable.

In Lab investigations, $\mathrm{Hb}$ was $10.6 \mathrm{~g} \%$, TLC 5,600/ $\mathrm{mcL}$ and Platelets 2,45,000 /mcL. BT, CT and PT were within normal range. ECG showed atrial fibrillation

Correspondence: $\operatorname{Dr}$ Khus Raj Dewan, Department of Gastroenterology, College of Medical Sciences Teaching Hospital, Bharatpur, Nepal. Email: dewansantosh@yahoo.com 
with controlled heart rate. Chest X-ray PA and left lateral views showed massive cardiomegaly with cardiothoracic ratio 0.74 (Figure $1 \& 2$ ). Transthoracic echocardiography revealed severe MS (MVA: $1 \mathrm{~cm} 2)$, severe MR, AS, TR with severe PAH: (RVSP: $141 \mathrm{~mm}$ Hg). LA diameter 87.9 mm. LVEF: 56\% (Figure 3 \& 4).

Upper GI Endoscopy showed narrowing of the lumen without evidence of obstruction and erosive oesophageal ulcer secondary to friction of the adjacent wall due to left atrium each heart beat at the middle $1 / 3$ of esophagus. Stigmata of recent hemorrhage were also noted (Figure 5).

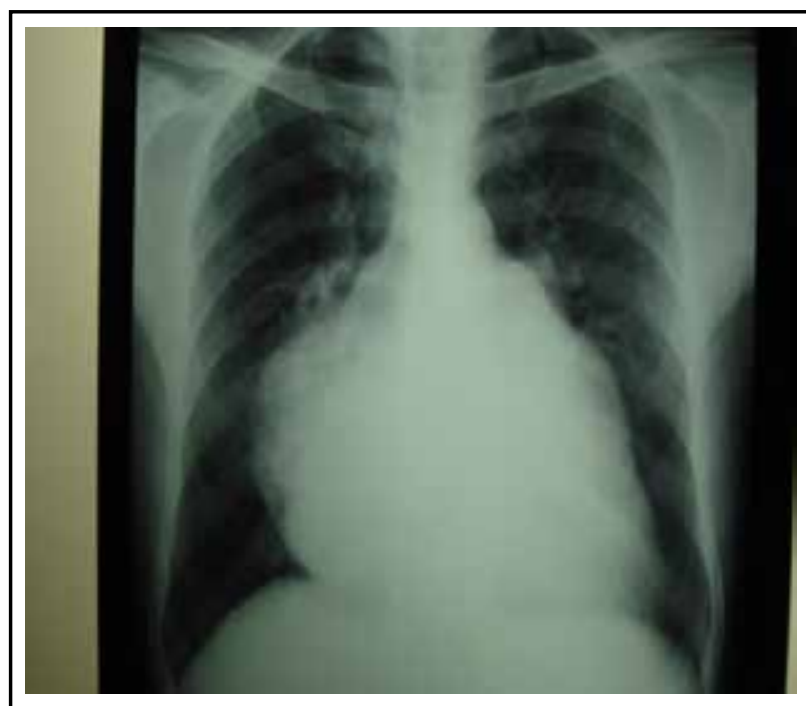

Figure 1. Chest $x$ ray PA view showing massive cardiomegaly.

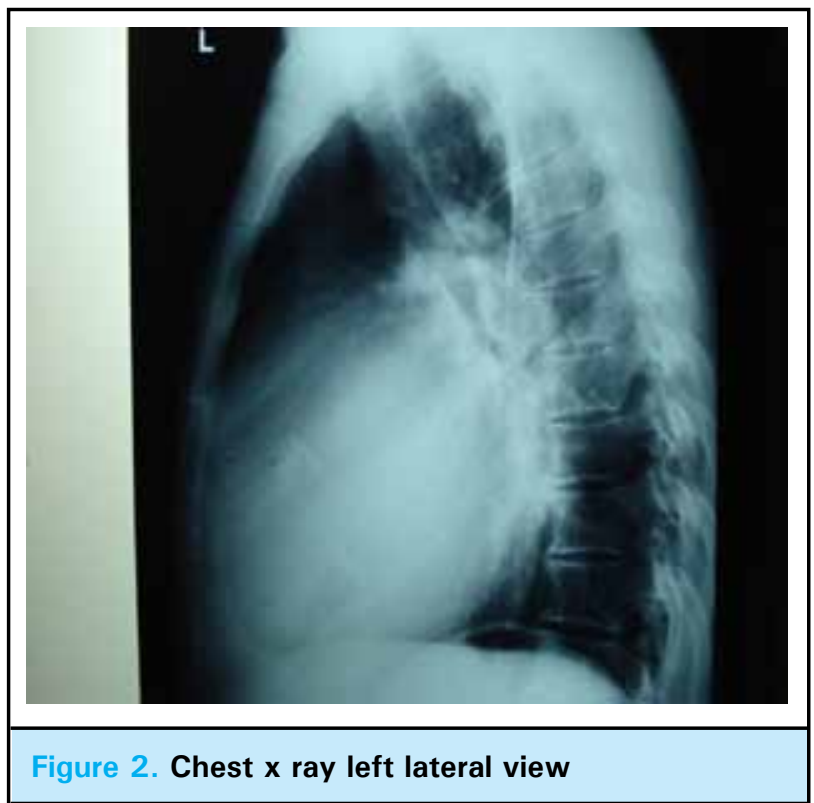

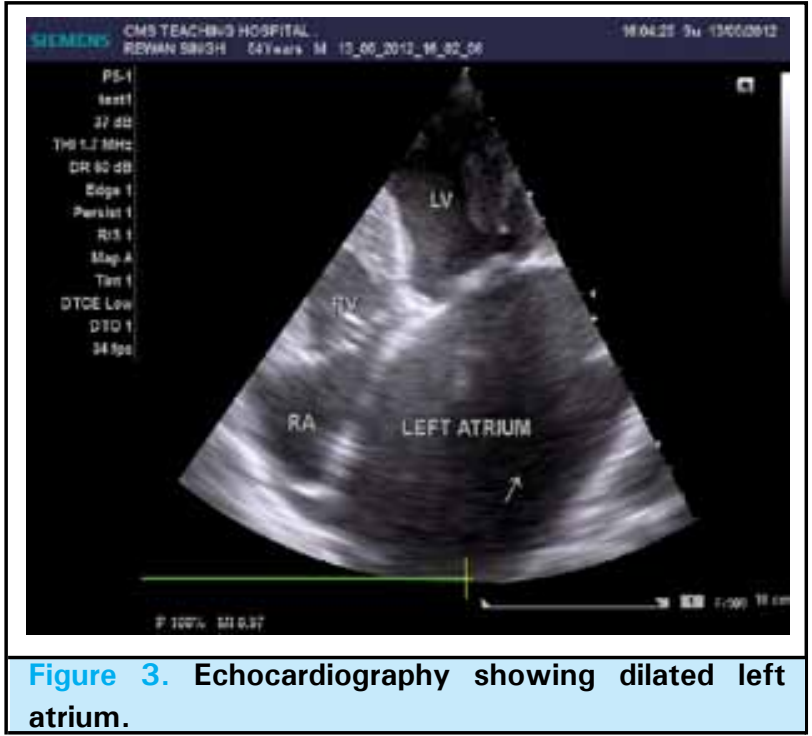

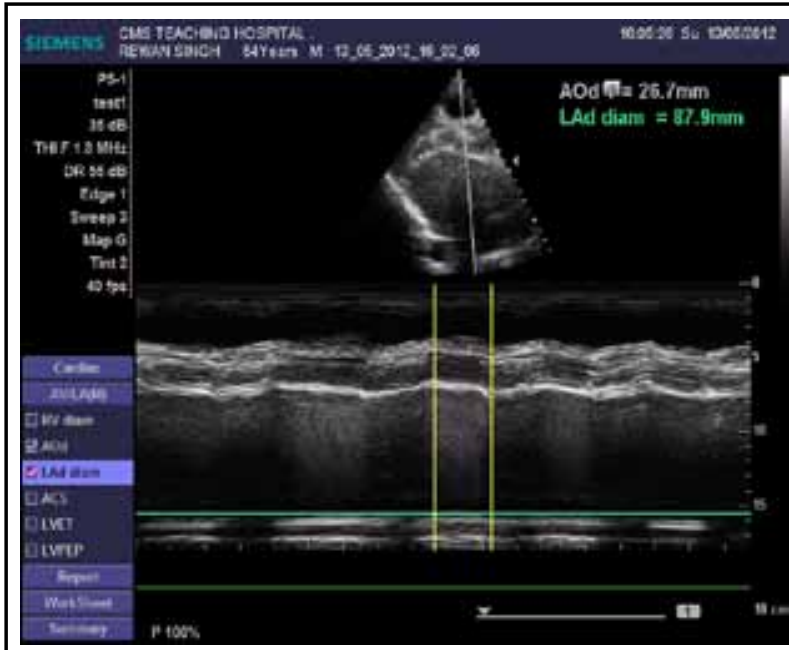

Figure 4. Echocardiography 2 D image measuring left atrium

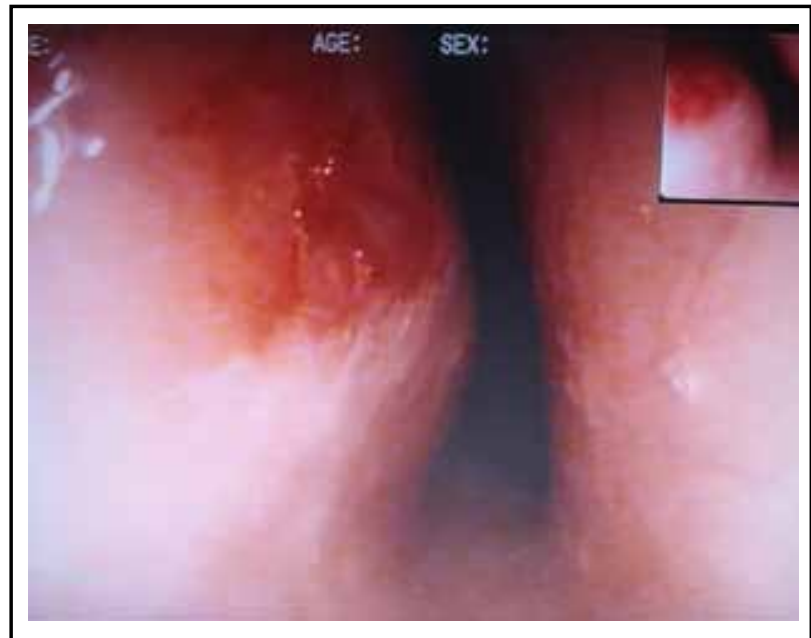

Figure 5. UGI Endoscopy showing narrowed oesophageal lumen with erosions. 


\section{DISCUSSION}

Enlargement of the left atrium is usually secondary to rheumatic mitral stenosis, mitral regurgitation or both. Most of the case reports of giant left atrium are due to rheumatic pathology which is a common health problem in the developing countries. 1,2,3 But there are few reports with a non-rheumatic etiology. Phua et $\mathrm{al}^{4}$ reported giant left atrium due to mitral valve prolapse. Yuksel et $\mathrm{al}^{5}$ reported giant left atrium due to hypertrophic cardiomyopathy and Cheng et al ${ }^{6}$ described its association with cardiac amyloidosis, causing a restrictive cardiomyopathy.

Hurst defined a giant left atrium as one that touches the right lateral side of the chest wall on chest xray. ${ }^{7}$ Isomura et al 8 defined it as left atrium of greater than 6 $\mathrm{cm}$ diameter while Kothari et $\mathrm{al}^{9}$ defined as greater than $8 \mathrm{~cm}$. Piccoli et $\mathrm{al}^{10}$ defined the giant left atrium as a cardio-thoracic ratio on CXR of more than 0.7 combined with a left atrial anterior-posterior diameter of more than $8 \mathrm{~cm}$ on transthoracic echocardiography. In developing countries where rheumatic heart disease is common, Ates et al suggested giant left atrium to be diagnosed when it is more than $10 \mathrm{~cm}$ in diameter while reporting a giant left atrium of $18.7 \mathrm{~cm} .{ }^{11}$ Giant left atrium is a rare entity in developed countries in the modern era. ${ }^{12}$ In our case report the left atrium diameter was 87.9 $\mathrm{mm}$ and the cardiothoracic ratio in PA view chest xay was 0.74

Enlarged left atrium not only gives haemodynamic consequences but it also produces compression and displacement of neighboring structures. In 1949, Daley and Franks ${ }^{13}$ described the clinical features and anatomical consequences of giant left atrium such as dysphagia from oesophageal displacement or compression against the vertebrae, compression of bronchi leading to complete obstruction of a lobar bronchus, especially the left lower and the middle lobar bronchi, compression of the left recurrent laryngeal nerve and very rarely even erosion of the spine. Thromboembolism is also a common complication of giant left atrium which was often mistaken for mediastinal tumor before the use of echocardiography. ${ }^{14}$ In 1969, Le Roux and Williams ${ }^{15}$ were the first to report oesophageal compression against the vertebrae by massive left atrial enlargement causing oesophageal erosion with upper gastrointestinal bleeding and death from haemorrhage. No other reports are available regarding giant left atrium causing compression and erosion of the oesophagus producing upper gastrointestin al bleeding.

Treatment of this group of patients is to correct the mitral valve abnormalities, decrease the left atrial size, to treat compression manifestations, to prevent thromboembolism and revert atrial fibrillation to normal sinus rhythm. Left atrial plication has also been recommended by some authors. ${ }^{16}$ In our case, there was severe mitral stenosis with advanced pulmonary hypertension in which operation was not possible due to high mortality risk. We aimed to report this case because there are several reports of giant left atrium but producing upper gastrointestinal bleeding due to compression of oesophagus and erosion is very rare. The patient was discharged home on medical management after recovery.

\section{REFERENCES}

1. Goldenberg G, Eisen A, Weisenberg N, Amital H. Giant left atrium. IMAJ. 2009;11:641

2. Tung R, DeSanctis R. Giant left atrium. NEJM. 2004;351:1437

3. Ates M, Sensoz Y, Abay G, Akcar M. Giant left atrium with rheumatic mitral stenosis. Texas Heart Inst J. 2006;33:389-91

4. Phua GC, Eng PC, Lim SL, Chua YL. Beyond Ortner's syndrome - Unusual pulmonary complications of the giant left atrium. Ann Acad Med. 2005;34:642-5.

5. Yuksel UC, Kursaklioglu H, Celik T. Apical hypertrophic cardiomyopathy with giant left atrium. Arq Bras Cardiol. 2007;88:e47.

6. Cheng Z, Fang Q, Liu Y. Cardiac amyloidosis with giant atria. Heart. 2010;96:1820

7. Hurst W. Memories of patients with a giant left atrium. Circulation. 2001;104:2630-1.

8. Isomura T, Hisatomi K, Hirano A, Maruyama H, Kosuga K, Ohishi K. Left atrial plication and mitral valve replacement for giant left atrium accompanying mitral lesion. J Card Surg 1993;8:365-70
9. Kothari AA, Kothari KA. A giant left atrium. J PostGrad Med. 2005; 51: 49-50.

10. Piccoli GP, Massini C, Di Eusanio G, Ballerini L, Tacobone G, Soro A, et al. Giant left atrium and mitral valve disease: Early and late results of surgical treatment in 40 cases. J Cardiovasc Surg. 1984;25:328-36.

11. Ates M, Sensoz Y, Abay G, Akcar M. Hugh Left Atrium. Eur J Gen Med 2006; 3(2):90-92

12. Krishnamoorthy KM. $100 \%$ Cardiothoracic ratio. Texas Heart Inst J. 2001;28:334-5.

13. Daley R,Franks R. Massive dilatation of the left auricle. Quart. J. Med.1949;18:81.

14. Schvartzman PR, White RD. Giant left atrium. Circulation. 2001;104:e28-9.

15. Le Roux BT, Williams MA. Dysphagia megalatriensis. Thorax.1969;24:603.

16. Tonguc E, Kestelli M, Ozsoyler I, Yilik L, Yilmaz A, Ozbek C et al. Limit of indication for plication of Giant Left Atrium. Asian Cardiovasc Thorac Ann. 2001; 9:24-6. 